\title{
Simultaneous in vivo confocal reflectance and two-photon retinal ganglion cell imaging based on a hollow core fiber platform
}

Tschackad Kamali

Jörg Fischer

Spring Farrell

William H. Baldridge

Gerhard Zinser

Balwantray C. Chauhan 


\section{Simultaneous in vivo confocal reflectance and two-photon retinal ganglion cell imaging based on a hollow core fiber platform}

\author{
Tschackad Kamali, ${ }^{a, *}$ Jörg Fischer, ${ }^{a}$ Spring Farrell,, \\ William H. Baldridge, ${ }^{\mathrm{b}, \mathrm{d}, \mathrm{e}}$ Gerhard Zinser, ${ }^{\mathrm{a}}$ and \\ Balwantray C. Chauhan ${ }^{\mathrm{b}, \mathrm{c}, \mathrm{e}}$ \\ aHeidelberg Engineering $\mathrm{GmbH}$, Heidelberg, Germany \\ ${ }^{b}$ Dalhousie University, Retina and Optic Nerve Research Laboratory, \\ Halifax, Nova Scotia, Canada \\ 'Dalhousie University, Physiology and Biophysics, Halifax, \\ Nova Scotia, Canada \\ dDalhousie University, Medical Neurosciences, Halifax, Nova Scotia, \\ Canada \\ eDalhousie University, Ophthalmology and Visual Sciences, Halifax, \\ Nova Scotia, Canada
}

\begin{abstract}
We have developed a compact hollow core fiber (HCF)-based imaging platform capable of simultaneous in vivo confocal reflectance and two-photon imaging through the mouse pupil. We demonstrate the performance of this platform by imaging retinal ganglion cells (RGCs) in which the fluorophores YFP and GCaMP3 are expressed in Thy1-YFP-16 and Thy1-GCaMP3 transgenic mice, respectively. Confocal reflectance images of the mouse retina served as a reference for the simultaneous acquisition of the two-photon signals that clearly showed RGCs with single-cell resolution. The use of an HCF platform makes the system compact with future application in the longitudinal investigation into the structure and function of healthy and diseased RGCs. (0) The Authors. Published by SPIE under a Creative Commons Attribution 3.0 Unported License. Distribution or reproduction of this work in whole or in part requires full attribution of the original publication, including its DOI. [DOI: 10.1117/1.JBO.23.9.091405]
\end{abstract}

Keywords: two-photon microscopy; fluorescence microscopy; biomedical optics; photonics; ophthalmic devices.

Paper 170767SSR received Nov. 28, 2017; accepted for publication Mar. 5, 2018; published online Mar. 26, 2018.

Two-photon excitation (TPE) fluorescence imaging is a powerful emerging tool in biomedical applications, providing high penetration depth and inherent three-dimensional (3-D) sectioning at the subcellular level. ${ }^{1-3}$ The retina is the only tissue in which single neurons can be imaged optically and noninvasively due to the high transparency of the preretinal tissues. ${ }^{4,5}$ TPE with infrared light (IR) is particularly well suited for in vivo retinal imaging. Reporter molecules in cell bodies can be excited with IR light to allow differential activation of rod and cone

*Address all correspondence to: Tschackad Kamali, E-mail: tschackad kamali@ heidelbergengineering.com photoreceptors by wavelengths in the visually sensitive range to evoke responses in the retina. ${ }^{6-9}$ An additional advantage of TPE is reduced phototoxicity. ${ }^{8}$ TPE fluorescence imaging enables the study of functional physiological processes, which, in combination with in vivo ophthalmoscopy, represent powerful imaging techniques that are well suited for noninvasive in vivo retinal imaging. Applications include longitudinal tracking of disease progression, for example, in optic neuropathies in which retinal ganglion cells (RGCs), the output neurons from the eye to the brain, are lost.

To date, there are relatively few reports published on the applicability of in vivo TPE fluorescence intensity imaging in the retina. ${ }^{10,11}$ Systems used thus far are bulky and complex and require sophisticated adaptive optics (AO) to compensate for the wavefront errors introduced by the lens and cornea, hence achieving a tightly focused spot and increased TPE signal rate. Recently, a non-AO two-photon mouse retinal imaging system was described $;{ }^{9}$ however, a water-immersion contact microscope objective lens was used for the focusing optics and the imaging required surgical fixation of the mouse skull.

In this work, we demonstrate, for the first time to our knowledge, a system based on a hollow core fiber (HCF) that is capable of simultaneous in vivo confocal reflectance and two-photon imaging of RGCs through the mouse pupil without the use of AO. One strategy to reduce the need for $\mathrm{AO}$ in the device presented here is the use of eye tracking software (Heidelberg Engineering, Heidelberg, Germany) that is currently implemented in clinical confocal scanning laser devices for ophthalmoscopy and tomography. ${ }^{12}$ The real-time eye tracking software enables prolonged signal collection from the same spot, which is critical at low signal levels and in suboptimal light focusing conditions. The simultaneous acquisition of confocal reflectance and two-photon images colocalizes precisely the retinal location, where the two-photon recordings originate. Together, these features provide images with single cell resolution along with wider field fundus images. The use of an HCF for laser delivery allows our system to be split into a compact application unit (dashed blue area, Fig. 1) and a separate laser unit that can be placed on a nearby optical bench (gray shaded area, Fig. 1) without introducing noticeable pulse broadening, which is limited to approximately $-200 \mathrm{fs}^{2} / \mathrm{m}$ in our system. The application unit itself consists of a modified commercial scanning laser ophthalmoscope and an optical coherence tomography unit (Spectralis, Heidelberg Engineering) used routinely in clinical practice.

The Ti:Sapphire light source (Chameleon Ultra II, Coherent, Santa Clara, California) was tuned to a center wavelength of $930 \mathrm{~nm}$ for confocal reflectance imaging as well as two-photon signal generation. The laser had a repetition rate of $80 \mathrm{MHz}$ and produced pulses of $140 \mathrm{fs}$ duration with an output power of $1.6 \mathrm{~W}$ at $930 \mathrm{~nm}$. A half-wave plate (HWP) and a polarization beam splitter were used for power adjustment. The group delay dispersion of the complete optical setup amounted to $\sim 7000 \mathrm{fs}^{2}$, which was compensated for by a femtosecond pulse compressor based on prism pairs (FSPC, Thorlabs, Newton, New Jersey). The pulse duration at the sample position was measured with an autocorrelator (Mini USB PMT NIR, APE, Berlin, Germany), which confirmed approximately transform-limited pulses of 154 fs. A CCD camera (FireflyMV, FLIR, Wilsonville, Oregon) was used to measure the beam profile at the sample position. Both second-order autocorrelation measurement and the Gaussian beam profile measurements are shown in Fig. 2. 


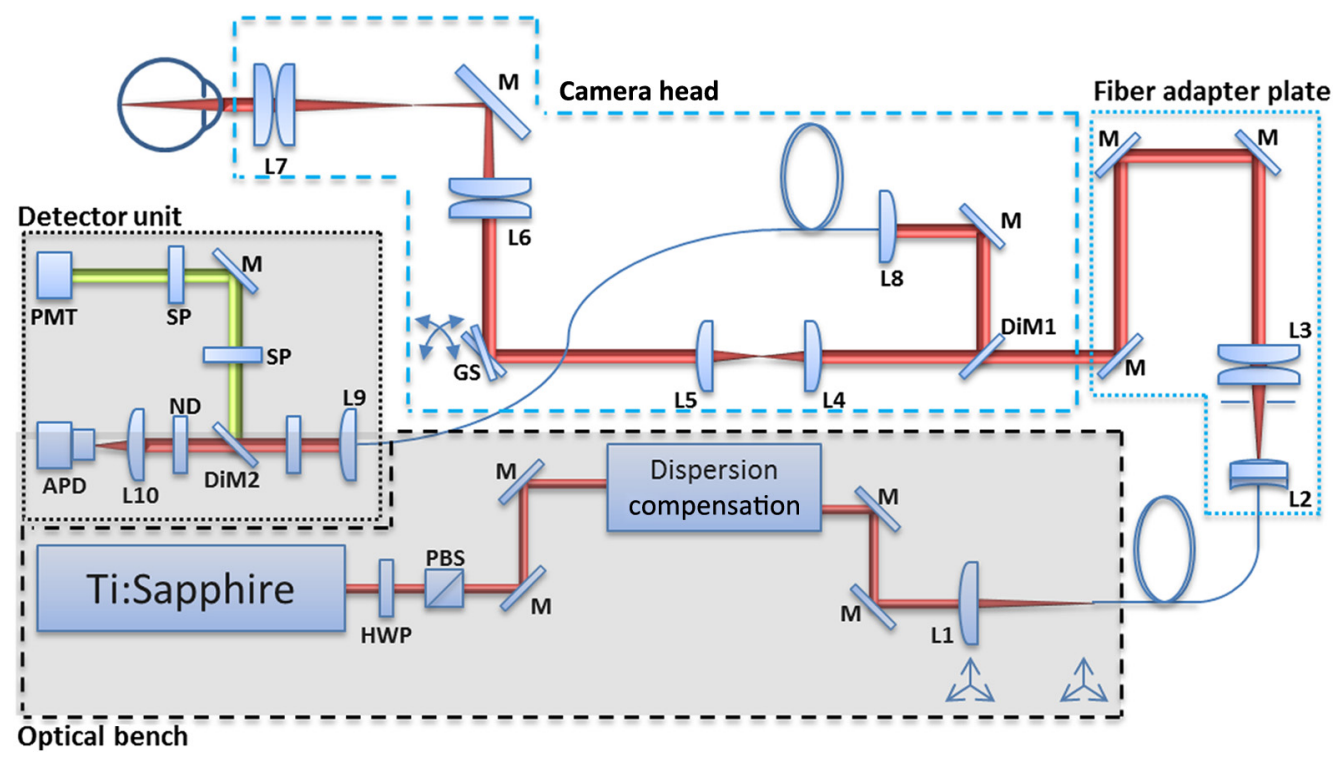

Fig. 1 Simultaneous confocal reflectance and two-photon imaging setup: the output of the Ti:Sapphire laser is adjusted with an HWP and a polarizing beam splitter. After dispersion compensation with prism pairs, the light is coupled into an HCF with a lens (L1), where both the HCF and L1 are mounted on separate three-dimensional translation stages. The output side of the fiber is connected via an FC connector to the fiber adapter plate (blue dotted line) that is attached to the modified Heidelberg Engineering Spectralis camera head (blue dashed line). The divergence of the fiber output is increased with a negative lens (L2) before coupling it to a double achromatic lens (L3). Two lenses of equal focal length (L4 and L5) were used for fine focal readjustments. Lateral scanning was performed with galvanometric scanners (GS), where the pivot point was imaged on the mouse pupil with a scan and tube lens (L6 and L7, respectively). A dichroic beam splitter (DIM1) in the camera head reflected the signal that was coupled to a multimode fiber with a lens (L8). In the detection unit (black dotted line), the output of the fiber was collimated with a lens (L9), and a dichroic mirror (DIM2) separated the fluorescence signal from the reflectance signal. In the reflectance path, an ND filter is used for attenuation. A lens (L10) focuses the light on an avalanche photodetector. The fluorescence signal path contains another short pass blocking filter (SP) before detection with a photomultiplier tube. Steering mirrors (M); shaded box indicates optical components placed on an optical bench; black dashed line represents the excitation path while black dotted line represents the detection path.

After dispersion compensation, the laser beam was coupled to a 2-m HCF (GLOphotonics, Limoges, France) with a 45-mm focal length achromatic lens (AC254-045-B, Thorlabs) with which a coupling efficiency of $\sim 89 \%$ was achieved. The

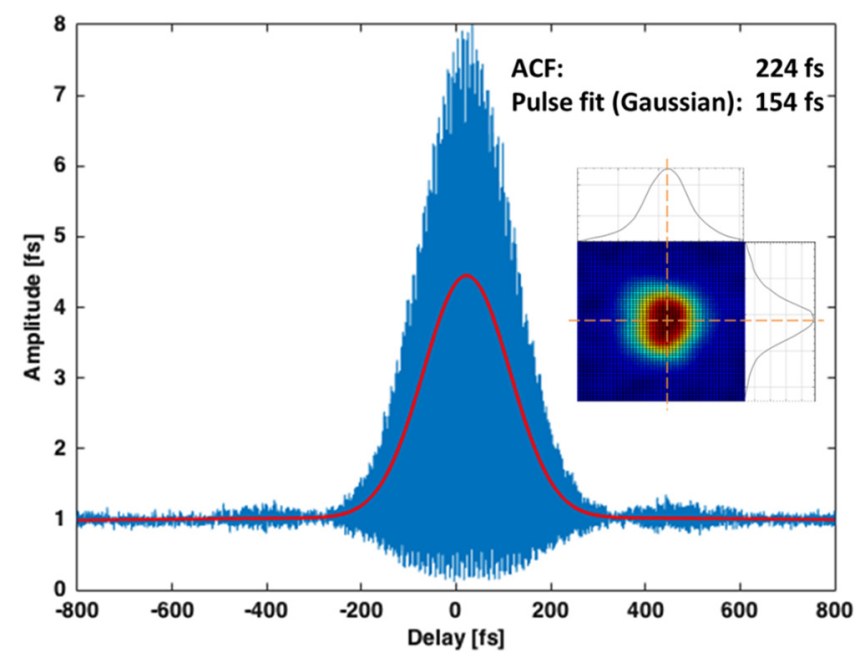

Fig. 2 Pulse measurement at sample position with interferometric autocorrelation showing an approximately transform-limited pulse. Inset shows the beam profile of the laser measured with a CCD camera at the sample position with vertical and horizontal line beam profiles. coupling lens was mounted on a $25-\mathrm{mm} X Y Z$ translation stage (PT3, Thorlabs) and the fiber on a 3 -axis microblock stage (MBT616D, Thorlabs).

The fiber output was coupled via FC connector to the fiber adapter plate that was mounted to the camera head of the modified Spectralis unit. The divergence of the output beam from the fiber was increased with a $-6.0-\mathrm{mm}$ focal length, biconcave, negative lens (LD2746-B, Thorlabs) to avoid the use of a longer focal length collimator before it was collimated with an achromatic doublet lens with a focal length of $25 \mathrm{~mm}$ (AC 127-025-B, Thorlabs). Two customized lens pairs of equal focal length, $f=$ $20 \mathrm{~mm}$ (L4, L5 in Fig. 1), were integrated to enable finer focus adjustment in the axial plane. Horizontal and vertical beam scanning was performed with the standard Spectralis scan unit. In combination with a customized $50-\mathrm{mm}$ focal length scan lens, an intermediate image field of $5 \times 5 \mathrm{~mm}^{2}$ was produced. A customized 16-mm focal length tube lens translated the intermediate image field to a field of view of $\sim 17.5 \mathrm{deg}$ while achieving a beam size of $\sim 2.2 \mathrm{~mm}$ (overfilling the dilated mouse pupil). Both reflectance and two-photon fluorescence signal were repassed through the scanning unit, resulting in a stationary, descanned light beam. A dichroic mirror (FF735Di02, Semrock, Rochester, New York) was used to couple the fluorescence and reflectance signal into the detection branch, consisting of an 40-mm focal length achromatic lens and a $100-\mu \mathrm{m}$ multimode fiber, which guides the signal light to 

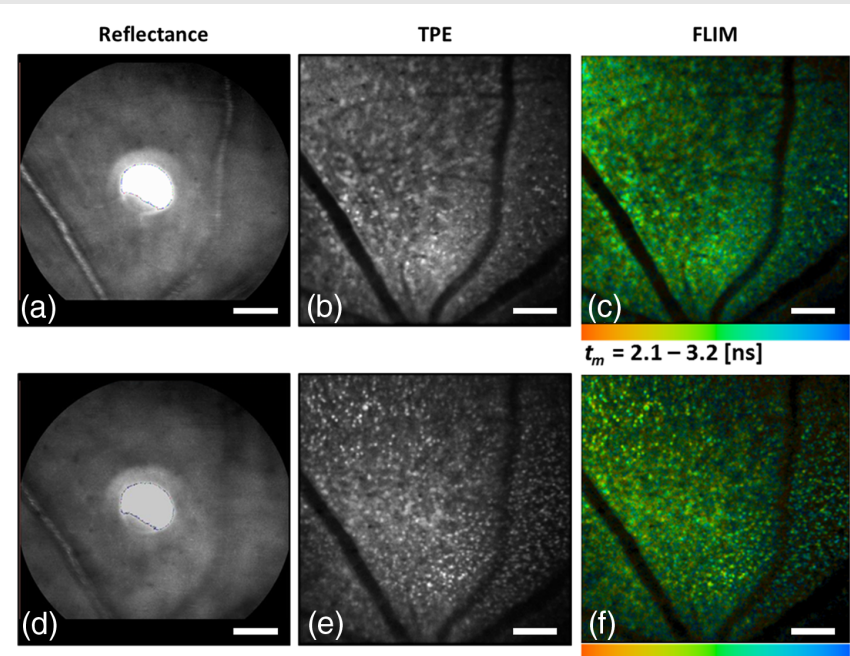

$t_{m}=2.1-3.2[n s]$

$t_{m}=1.8-3[\mathrm{~ns}]$

Fig. 3 In vivo confocal reflectance and two-photon images of the retina of a Thy1-YFP-16 mouse. (a) Confocal reflectance image showing mouse fundus. (b) Simultaneously obtained TPE fluorescence image at same transverse and axial position as in (a). (c) FLIM of (b) with scale bar of fluorescence lifetime $\left(t_{m}\right)$. (d) Confocal reflectance image at same transverse position as in (a) but at different depths. (e) Simultaneously obtained two-photon image clearly showing RGCs. (f) FLIM of (e). Images were obtained with power levels $\sim 10 \mathrm{~mW}$ and exposure times of 2 to $3 \mathrm{~min}$. Scale bar, $100 \mu \mathrm{m}$.

the external detection unit. The fiber output was collimated with a 12-mm focal length lens and a second dichroic mirror (FF735Di02, Semrock) separated the visible fluorescence light from the near infrared (NIR) reflectance light. The reflectance light was further attenuated with a neutral density (ND) filter and focused on an avalanche photodiode (RCA, New York City) with a $20-\mathrm{mm}$ focal length customized achromatic lens. In the fluorescence signal path, a short-pass filter (FF01-720/SP-25, Semrock) was used to remove any leakage from the excitation light before being focused by a $100-\mathrm{mm}$ focal length achromatic lens (47-972, Edmund Optics, Barrington, New Jersey) onto the photon counting detector (HPM-100-50, Becker-Hickl, Berlin, Germany) connected to a time-correlated single photon counting (TCSPC) module (SPC-150, Becker-Hickl). All imaging was performed with a horizontal line scan rate of $8 \mathrm{kHz}$ and a pixel clock of $10 \mathrm{MHz}$. Confocal reflectance images were digitized at a resolution of $768 \times 768$ pixels with a frame rate of $\sim 9 \mathrm{~Hz}$. Fluorescence images were digitized at a $256 \times 256$ pixel resolution (by binning the corresponding signal to superpixels) and were averaged over 2 to $3 \mathrm{~min}$. Customized software for real-time eye tracking (Heidelberg Engineering) was used for imaging. The confocal reflectance image served as a reference for the two-photon fluorescence signals, whose acquisition took place after correct positioning and localization. In this manner, each detected fluorescence photon could be assigned to the corresponding pixel from the reflectance image.

Two mouse strains, Thy1-YFP-16 [B6.Cg-Tg(Thy1-YFP) $16 \mathrm{Jrs} / \mathrm{J} ; 6$ months old male, 35 to $40 \mathrm{~g}$, The Jackson Laboratory, Bar Harbor, Maine] and Thy1-GCaMP3 [B6.Cg-Tg(Thy1GCaMP3)6Gfng/J; 6 months old male, 35 to $40 \mathrm{~g}$, The Jackson Laboratory], were used for imaging. Mice were anesthetized with ketamine $(100 \mathrm{mg} / \mathrm{kg})$ and xylazine $(10 \mathrm{mg} / \mathrm{kg})$ by intraperitoneal injection. Pupils were dilated with one drop of $1 \%$ tropicamide (Mydriacyl, Alcon Laboratories, Mississauga, Ontario, Canada) and one drop of $2.5 \%$ phenylephrine
(Mydfrin, Alcon Laboratories). After dilation, a 3.2-mm plano contact lens (Cantor and Nissel, Brackley, United Kingdom) was placed on the cornea to maintain corneal hydration and compensate for most of the nonspherical refractive errors arising from the corneal surface. During imaging, the mouse was placed on a custom-built translation stage and a bite bar was used to stabilize the head for camera alignment. All experimental procedures followed the guidelines of the Canadian Council on Animal Care, and protocols were approved by the Dalhousie University Committee on Laboratory Animals.

Fluorescence lifetime maps, obtained with fluorescence lifetime imaging (FLIM), deliver additional information about cell health. ${ }^{13-15}$ FLIM is mainly concentration independent and measures the average duration a molecule remains in an excited state. This duration is unique, providing a molecular fingerprint. ${ }^{16}$ Changes in fluorescence lifetime reflect changes in cellular environment, such as temperature, $\mathrm{pH}$, ion, and oxygen concentration. ${ }^{17,18}$

First, we performed imaging at two different retinal depths in Thy 1-YFP-16 mice. The simultaneous acquisition of the confocal reflectance image as well as the TPE image are shown in Fig. 3.

Focused at the level of the retinal nerve fiber layer where axons of RGCs are located, the confocal reflectance images visualize the mouse fundus [Fig. 3(a)], whereas the TPE images show RGCs [Fig. 3(b)]. For each TPE pixel, the mean fluorescence lifetime $\left(t_{m}\right)$ was determined and displayed in a colorcoded FLIM map with a decay time range from $2.1 \mathrm{~ns}$ (red) to $3.2 \mathrm{~ns}$ (blue) [Fig. 3(c)]. The same imaging was performed $\sim 20-\mu \mathrm{m}$ deeper but at the same lateral position. This axial position was approximately at the level of the RGC somas [Figs. 3(e) and 3(f)]. Although RGCs are clearly visualized in the Thy1YFP-16 mouse strain [Figs. 3(b) and 3(e)], the acquisition of static fluorescence intensity measurements of RGCs does not deliver sufficient information to discriminate functional from nonfunctional RGCs. ${ }^{12}$ Previous work from our group has shown that, after experimental optic nerve injury, some RGCs expressing GCaMP3, a calcium indicator whose dynamics are related to changing calcium levels during neuronal action potentials, do not respond to a stimulus. ${ }^{19}$ Dynamic fluorescence intensity imaging of these markers enables probing of cellular function of individual RGCs in response to physiologic stimuli.

Next, to determine whether imaging calcium dynamics with GCaMP3 was feasible with TPE fluorescence imaging, we imaged RGCs in the Thy1-GCaMP3 mouse strain. We performed imaging at two different power levels and integration times to determine if sufficient fluorescence signals could be generated to visualize individual RGCs (Fig. 4). Even at low-power levels, (close to the human use safety threshold), ${ }^{9}$ individual RGCs were clearly visible. The quantity of fluorescence photons detected was too low to calculate an additional lifetime map.

FLIM measurements as presented in [Figs. 3(c) and 3(f)] have the potential to provide critical and complementary information about cell status ${ }^{20,21}$ and differentiate among RGC subtypes with different levels of vulnerability to damage. This work is currently in progress.

In summary, this non-AO TPE imaging platform, based on an $\mathrm{HCF}$, acquired in vivo images of RGCs in transgenic mice expressing YFP and GCaMP3 fluorescence markers. Additional fluorescence lifetime maps of YFP were obtained, showing that in vivo FLIM can be used to acquire complementary information. Lifetime maps of GCaMP3 were not possible due to low photon count. However, since RGCs are clearly visible at low power 

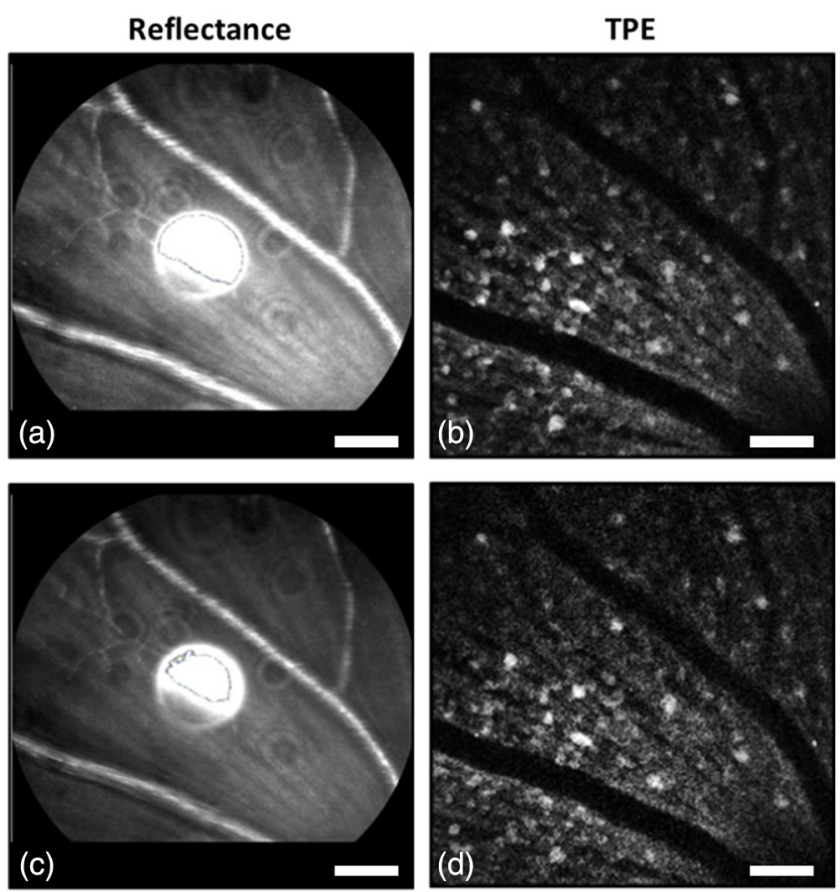

Fig. 4 In vivo confocal reflectance and TPE fluorescence images of the retina of a Thy1-GCaMP3 mouse at different power levels and integration times. (a) Confocal reflectance image showing the mouse fundus with $\sim 10-\mathrm{mW}$ laser power. (b) Two-photon image acquired simultaneously with (a) showing individual GCaMP3 expressing RGCs; power $\sim 10-\mathrm{mW}$ and $\sim 3$ - min exposure time. (c) and (d) Reflectance and two-photon images, respectively, at the same lateral and axial location as (a) but with lower laser power $(\sim 3 \mathrm{~mW})$; exposure time $\sim 3.5 \mathrm{~min}$. Scale bar, $50 \mu \mathrm{m}$.

levels, FLIM with faster detectors could be used for dynamic intensity imaging and provide a lifetime map. Since FLIM is mainly independent of fluorophore concentration, it might offer advantages for longitudinal studies since the measurement is less dependent on the optical quality and expression levels of a reporter molecule. ${ }^{16,22}$ Future work will involve the implementation of a NIR laser diode to separate the light beams for confocal reflectance imaging and TPE.

\section{Disclosures}

T. Kamali, J. Fischer, and G. Zinser are affiliated with Heidelberg Engineering. B. Chauhan receives research support, but no royalty or honoraria, from Heidelberg Engineering. S. Farrell and W. Baldridge have no relevant disclosures.

\section{Acknowledgments}

The authors gratefully acknowledge funding from the Atlantic Canada Opportunities Agency Atlantic Innovation Fund (Grant No. 197809)

\section{References}

1. W. Denk, J. H. Strickler, and W. W. Webb, "Two-photon laser scanning fluorescence microscopy," Science 248(4951), 73-76 (1990).

2. F. Helmchen and W. Denk, "Deep tissue two-photon microscopy," Nat. Methods 2(12), 932-940 (2005).
3. K. Svoboda and R. Yasuda, "Principles of two-photon excitation microscopy and its applications to neuroscience," Neuron 50(6), 823-839 (2006).

4. Y. Imanishi, K. H. Lodowski, and Y. Koutalos, "Two-photon microscopy: shedding light on the chemistry of vision," Biochemistry 46(34), 9674-9684 (2007).

5. J. Dillon et al., "Transmission spectra of light to the mammalian retina," Photochem. Photobiol. 71(2), 225-229 (2000).

6. D. C. Gray et al., "In vivo fluorescence imaging of primate retinal ganglion cells and retinal pigment epithelial cells," Opt. Express 14(16), 7144-7158 (2006).

7. J. I. W. Morgan et al., "Light-induced retinal changes observed with high-resolution autofluorescence imaging of the retinal pigment epithelium," Invest. Ophthalmol. Visual Sci. 49(8), 3715-3729 (2008).

8. R. K. P. Benninger and D. W. Piston, "Two-photon excitation microscopy for the study of living cells and tissues," in Current Protocols in Cell Biology, J. S. Bonifacino et al., Eds., pp. 1-24, John Wiley \& Sons, Inc., Hoboken, New Jersey (2013).

9. A. S. Bar-Noam, N. Farah, and S. Shoham, "Correction-free remotely scanned two-photon in vivo mouse retinal imaging," Light 5(1), e16007 (2016).

10. R. Sharma et al., "In vivo two-photon imaging of the mouse retina," Biomed. Opt. Express 4(8), 1285 (2013).

11. G. Palczewska et al., "Noninvasive two-photon microscopy imaging of mouse retina and retinal pigment epithelium through the pupil of the eye," Nat. Med. 20(7), 785-789 (2014).

12. S. J. Langenegger, J. Funk, and M. Töteberg-Harms, "Reproducibility of retinal nerve fiber layer thickness measurements using the eye tracker and the retest function of spectralis SD-OCT in glaucomatous and healthy control eyes," Invest. Ophthalmol. Visual Sci. 52(6), 3338-3344 (2011).

13. P. I. H. Bastiaens and A. Squire, "Fluorescence lifetime imaging microscopy: spatial resolution of biochemical processes in the cell," Trends Cell Biol. 9(2), 48-52 (1999).

14. W. Becker, "Fluorescence lifetime imaging-techniques and applications," J. Microsc. 247(2), 119-136 (2012).

15. W. Becker et al., "Fluorescence lifetime imaging by time-correlated single-photon counting," Microsc. Res. Tech. 63(1), 58-66 (2004).

16. M. Y. Berezin and S. Achilefu, "Fluorescence lifetime measurements and biological imaging," Chem. Rev. 110(5), 2641-2684 (2010).

17. K. Suhling et al., "Fluorescence lifetime imaging (FLIM): basic concepts and some recent developments," Med. Photonics 27(Suppl. C), 3-40 (2015).

18. J. Horilova et al., "Fingerprinting of metabolic states by NAD(P)H fluorescence lifetime spectroscopy in living cells: a review," Med. Photonics 27(Suppl. C), 62-69 (2015).

19. S. N. Blandford et al., "Retinal characterization of the Thy1-GCaMP3 mouse after optic nerve transection," Invest. Ophthalmol. Visual Sci. 57(12), 607-607 (2016).

20. C. Dysli et al., "Fluorescence lifetime imaging ophthalmoscopy," Prog. Retinal Eye Res. 60, 120-143 (2017).

21. A. J. Walsh et al., "Fluorescence lifetime imaging of calcium flux in neurons in response to pulsed infrared light," Proc. SPIE 10069, 100691B (2017).

22. J. A. Feeks and J. J. Hunter, "Adaptive optics two-photon excited fluorescence lifetime imaging ophthalmoscopy of exogenous fluorophores in mice," Biomed. Opt. Express 8(5), 2483-2495 (2017).

Gerhard Zinser received his $\mathrm{MS}$ and $\mathrm{PhD}$ degrees in physics from the University of Heidelberg in 1979 and 1982, respectively. His research focused on the design and development of novel clinical diagnostic devices for eye care professionals, including confocal scanning laser ophthalmoscopy and tomography and optical coherence tomography and angiography. In 2016, The Ophthalmologist named him among the top 100 people in the field. Sadly, he passed away shortly after this manuscript was submitted.

Biographies for the other authors are not available. 